Original Paper http://ajol.info/index.php/ijbes http://indexmedicus.afro.who.int

\title{
Distribution et structure des communautés de vers de terre et leur corrélation avec des éléments traces métalliques (ETM) le long des bordures de l'autoroute du Nord en Côte-d'Ivoire
}

\author{
Mamadou TOURE ${ }^{1,2^{*}}$, Seydou TIHO ${ }^{1,2}$, N'guetta Moïse EHOUMAN $^{1,2}$ et \\ Kouakou Gains KPAN KPAN ${ }^{1,2}$ \\ ${ }^{1}$ UFR des Sciences de la Nature (SN), Université Nangui Abrogoua, 02 BP 801 Abidjan 02, Côte d'Ivoire. \\ ${ }^{2}$ Unité de Recherche en Ecologie et Biodiversité (UREB). Université Nangui Abrogoua, Côte d'Ivoire. \\ *Auteur correspondant ; E-mail: tourexham@yahoo.fr, Tel : +22507177698
}

\section{RESUME}

Les sols des bordures de routes constituent des réservoirs importants de polluants susceptibles de perturber la survie des organismes qui y vivent. La présente étude vise à analyser la distribution et la structuration des communautés de vers de terre et leur corrélation avec des éléments traces métalliques (ETM) suivant un gradient, le long des bordures de l'autoroute du Nord en Côte d'Ivoire. Les vers de terre et les ETM ont été échantillonnés à partir de monolithes de type « Tropical Soil Biology and Fertility », réalisés le long de 10 transects. Dix-neuf (19) espèces de vers de terre ont été collectées et 8 ETM identifiés. Il est ressortit que le long des bordures de l'autoroute, la densité totale et le nombre d'espèces de vers augmentent au fur et à mesure qu'on s'éloigne de la chaussée. Leur structuration est marquée par une prépondérance des espèces endogées par rapport aux espèces épigées. La corrélation entre les vers de terre et les ETM montre que les ETM expliquent la variabilité principale des communautés de vers de terre. Le thallium, le zinc, le nickel, le plomb et le cuivre sont les principaux polluants qui semblent influencer la survie des communautés des vers de terre. Parmi les espèces de vers collectées, Dichogaster bolaui, Dichogaster sp1, Dichogaster sp2, Millsonia ghanaensis sont négativement influencés par les ETM. Ces résultats mettent en évidence une possibilité de choix des vers de terre comme (i) indicateurs de la pollution en ETM et (ii) organismes biologiques cibles pour les études d'impact environnemental.

(C) 2017 International Formulae Group. All rights reserved.

Mots clés : Vers de terre, ETM, Autoroute, Côte-d'Ivoire.

\section{Distribution and structure of the earthworm community and their correlation with heavy metals along of the northward motorway's edges in Côte d'Ivoire}

\section{ABSTRACT}

Roadside soils are important reservoirs of pollutants that can disrupt the survival of living organisms. This study aims at analyzing the distribution and structuring of earthworm community and their correlation with heavy metals along a gradient of the northward motorway's edges in Côte d'Ivoire. The earthworms and heavy metals were sampled using TSBF (Tropical Soil Biology and Fertility) monoliths carried out along 10 
transects. Nineteen (19) earthworms' species were collected and 8 heavy metals were identified. It was found that along the highway edges, the total density and number of earthworms species increased as one moved away from the roadway. Their structuring is marked by a preponderance of endogeic species compared with the epigeic species. Analysis of the correlation between earthworms and heavy metals showed that heavy metals explain the main variability in earthworm communities. Thallium, zinc, nickel, lead and copper are the major pollutants that influence the survival of earthworm communities. Among the earthworm species collected, Dichogaster bolaui, Dichogaster sp1, Dichogaster sp2, Millsonia ghanaensis are negatively influenced by heavy metals. These results indicate an ability to use earthworms as (i) indicators of pollution caused by HM and (ii) target biological organisms of environmental impact assessment.

(C) 2017 International Formulae Group. All rights reserved.

Keywords: Earthworm, Heavy Metals, Motorways, Côte d'Ivoire.

\section{INTRODUCTION}

Dans de nombreux pays d'Afrique sub-saharienne, la démographie galopante, combinée à une urbanisation accélérée ont eu pour conséquence l'augmentation du trafic routier (Banque Mondiale, 2003). Ce trafic est par ailleurs marqué par l'utilisation de véhicules d'occasion importés, l'absence de contrôles techniques fiables des véhicules, ainsi que l'utilisation croissante des engins à deux roues ou de carburants de mauvaise qualité (N'Guessan, 2010; Public Eye inverstigation, 2016). Par conséquent, la concentration des substances polluantes, notamment, les éléments traces métalliques (ETM), augmente au niveau des sols des bordures de routes et d'autoroutes. Selon Bentum et al. (2010) et Durand (2003), les ETM engendrés par le trafic routier représentent une fraction importante de la pollution chronique des sols. En effet, ces substances sont d'abord émises vers l'atmosphère le long des axes routiers et autoroutiers, puis retombent sous forme de dépôts secs ou humides sur la voie et ses alentours (Durand, 2003). Suite à la non biodégradabilité de ces ETM, ils s'accumulent dans le sol, altèrent les processus biologiques naturels (Trombulak et Frissell, 2000) et peuvent entrer, par l'intermédiaire des végétaux, dans la chaîne trophique (Baby et al., 2010; Kihampa et Mwegoha, 2010; Xiong et al., 2014) et constituer une menace pour la santé animale et humaine (Jackson, 2009; Singh et al., 2010 ; Jolly et al., 2013). Les études menées par Panisset et al. (2003) ; WHO (2013) ont révélé que l'accumulation de ces substances dans l'organisme animal et humain entraîne de graves affections telles que, les maladies neurologiques, le cancer, l'ostéomalacie, l'hydrargyrisme, la néphrite tubulaire et les troubles de la croissance chez l'enfant.

Par ailleurs, des études réalisées sur les sols pollués en ETM ont révélé que la densité, la capacité de survie et la reproduction des organismes endogés tels que les vers de terre sont négativement affectés (Spurgeon et al., 2003 ; Lévêque et al., 2015). Ces organismes sont pourtant des acteurs très importants dans la vie et la fertilité des sols (Brown et al., 2000). Qualifiés «d'ingénieurs de l'écosystème », les vers de terre génèrent des changements directs ou indirects sur les ressources d'autres espèces, en impactant les compartiments biotiques et abiotiques (Hale, 2005). De ce fait, leur abondance spécifique dans les sols influence les invertébrés (termites, fourmis, nématodes etc.) (Lavelle et al., 2006), les microorganismes (Blouin et al., 2013) et la croissance des plantes (Huynh, 2009). Aussi, la présence des vers de terre dans le sol est synonyme d'un bon état de santé de celui-ci (Conder et al., 2001).

Dans la région Ouest Africaine, l'autoroute du Nord, en Côte d'Ivoire, est l'un des axes routiers ayant un trafic intense, avec une fréquence de plus de 8000 véhicules par jour (FER, 2016) car elle relie Abidjan (capitale économique) à de nombreuses villes de la sous-région, notamment, celles du Burkina-Faso, du Mali, de la Guinée, etc. Il est donc opportun d'étudier l'effet de ce trafic sur la diversité endogée, particulièrement 
celle des vers de terre qui sont d'excellents bioindicateurs, afin d'anticiper sur les conséquences des ETM sur la biocénose qui jalonne les bordures de routes et d'autoroutes de cette région. C'est dans cette optique que la présente étude a été réalisée. Elle vise à analyser la distribution et la structure des communautés de vers de terre ainsi que leur corrélation avec des ETM suivant un gradient de distance, le long des bordures de l'autoroute du Nord, en Côte d'Ivoire. Ce travail repose sur l'hypothèse suivante: la distribution et la structuration des vers de terre varient le long des bordures de l'autoroute en corrélation avec les ETM émanant du trafic routier.

\section{MATERIEL ET METHODES}

\section{Site d'étude}

Cette étude a été réalisée le long des bordures de l'autoroute reliant les districts d'Abidjan et de Yamoussoukro, respectivement, capitale économique et capitale politique de la Côte d'Ivoire. Cette autoroute, longue de $220 \mathrm{~km}$, a été subdivisée en deux tronçons dont l'ancien tronçon, reliant le district d'Abidjan et la ville de Singrobo (140 km de longueur), mis en service en 1982, et le nouveau tronçon, reliant la ville de Singrobo au district de Yamoussoukro $(80 \mathrm{~km}$ de longueur), mis en service en décembre 2013. Le nouveau tronçon n'étant pas encore officiellement inauguré au moment de l'exécution de la présente étude, les travaux ont été effectués le long de l'ancien tronçon (Abidjan-Singrobo), localisé entre les $5^{\circ} 18$ et $6^{\circ} 80$ de latitude Nord et $4^{\circ} 00$ et $5^{\circ} 50$ de longitude Ouest.

\section{Dispositif d'échantillonnage}

Le long du tronçon choisi (AbidjanSingrobo), sur une distance de $90 \mathrm{~km}$, dix transects linéaires, distants de $10 \mathrm{~km}$ les uns des autres, reliant les deux bords de l'autoroute, en passant par le terre-plein, ont été tracés perpendiculairement à la chaussée. Sur chacun des transects, 11 monolithes de type TSBF (Tropical Soil Biology and Fertility) modifié à cause de la grande taille des vers de terre $(50 \mathrm{~cm} \times 50 \mathrm{~cm}$ sur $30 \mathrm{~cm}$ de profondeur) ont été creusés. Cinq monolithes à équidistance de $50 \mathrm{~m}$ ont été creusés de part et d'autre de la chaussée tandis qu'un seul l'a été au niveau du terre-plein central (TPC). Les 05 monolithes ont été creusés sur un transect linéaire virtuel de $200 \mathrm{~m}$ de longueur. $\mathrm{Au}$ total, 110 monolithes ont été creusés, soit, 50 monolithes de part et d'autres de l'autoroute, et 10 monolithes au niveau du terre-plein central (Figure 1).

\section{Echantillonnage et identification des vers de terre \\ Les vers de terre ont été récoltés} manuellement dans les strates 0-10 cm, 10-20 $\mathrm{cm}$ et $20-30 \mathrm{~cm}$ selon la méthode de Lavelle (1978). Des échantillons de sol ont été également prélevés au niveau de chaque strate et mélangés pour en sortir un échantillon composite. Les vers de terre collectés ont d'abords été conservés dans des bocaux dûment étiquetés contenant du formol dilué à $4 \%$. Ensuite, les bocaux ont été transportés au laboratoire où une identification des espèces a été faite à l'aide des clés de détermination de Cszudi et Tondoh (2007), basées sur la morphologie (forme, taille, longueur des segments) et la pigmentation des individus.

\section{Détermination et quantification des ETM}

Les échantillons composites de sol prélevés au niveau des strates de fouille de chacun des monolithes échantillonnés, ont servi à déterminer et quantifier les différentes substances ETM. Ces substances ont été identifiées par la méthode de la Spectrométrie à Diffusion d'Energies (EDS), à partir d'un Microscope Electronique à Balayage (MEB FEG Supra 40 VP Zeiss), au Laboratoire du Département d'Analyse et de Recherche de la Société Nationale d'Opération Pétrolière de Côte d'Ivoire (PETROCI). Ainsi, l'échantillon de sol a été finement broyé dans un mortier en porcelaine puis tamisé avec un tamis de maille $250 \mu$. Dix (10) mg de la poudre obtenue après tamisage ont été prélevés et étalés sur un plot apprêté avec du carbone adhésif à double face. L'appareil effectue une mesure de l'énergie de transition des électrons au niveau des nuages 
électroniques des séries $\mathrm{K}$, $\mathrm{L}$ et $\mathrm{M}$ des atomes de l'échantillon. Ce qui permet d'identifier sa composition chimique. Les substances ainsi identifiées ont été quantifiées à l'aide d'un spectromètre d'absorption atomique SPECTRA 110 (VARIAN). Cette opération est réalisée après digestion de $0,5 \mathrm{~g}$ de chaque échantillon dans $5 \mathrm{ml}$ d'acide nitrique $\left(\mathrm{HNO}_{3}\right)$ à $65 \%, 2 \mathrm{ml}$ d'acide sulfurique $\left(\mathrm{H}_{2} \mathrm{SO}_{4}\right)$ à $98 \%$ et $1 \mathrm{ml}$ de peroxyde d'hydrogène $\left(\mathrm{H}_{2} \mathrm{O}_{2}\right)$ à $30 \%$.

\section{Analyses statistiques}

Les comparaisons de densité moyenne et d'espèces de vers de terre entre les points d'échantillonnage, le long des transects ont été effectuées, respectivement, à l'aide du test non paramétrique de Kruskal- Wallis grâce au logiciel STATISTICA 7.1 et les indices de similarités de Jaccard grâce au logiciel EstimateS 7.5. La structure des communautés de vers de terre et leur corrélation avec les substances ETM, ont été déterminées à partir de différentes méthodes d'ordination développées par Lebreton et al. (1988), notamment, l'analyse factorielle des correspondances (AFC) et l'analyse canonique des correspondances (ACC). En effet, cette dernière analyse est particulièrement adaptée à l'analyse de la relation entre les espèces et leur habitat (Ter Braak et Šmilauer, 2002). Le but de l'ordination canonique est de rechercher la variation principale observée dans la relation entre les espèces et l'environnement (Ter Braak et Šmilauer, 2002). Cette technique a été utilisée pour interpréter les données parce qu'en écologie des communautés et en écologie environnementale étaient surtout multivariées. Ces différentes ordinations ont été exécutées à l'aide du package ade4 du logiciel R. Un test de permutation de MonteCarlo a été appliqué pour déterminer la signification statistique des axes de l'ACC. Quant aux coefficients canoniques de corrélation, ils ont été utilisés pour déterminer les substances polluantes contribuant significativement à la construction des principaux axes canoniques.

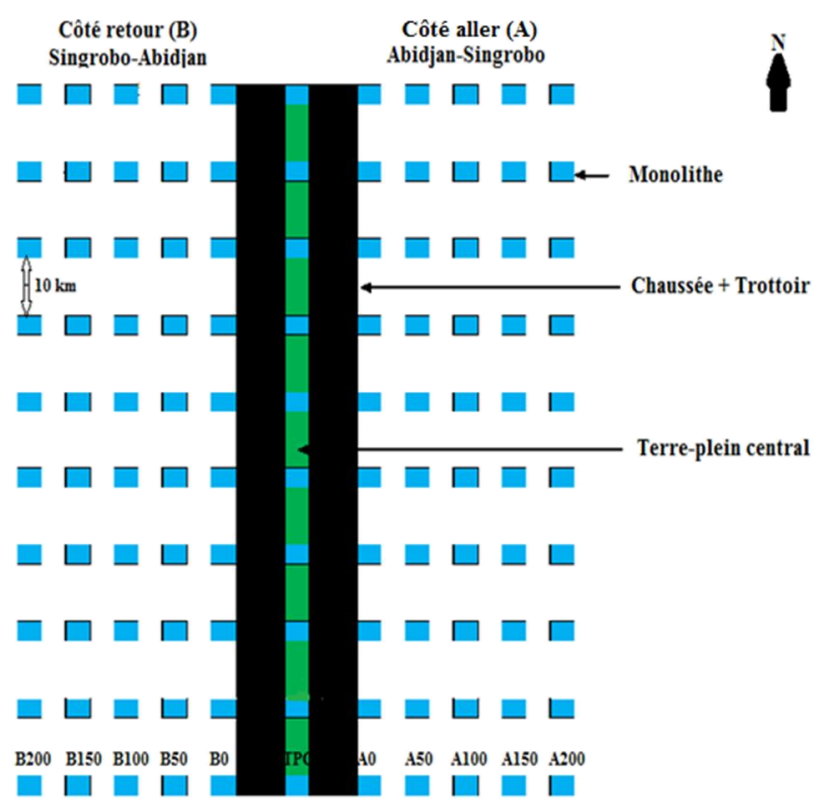

Figure 1 : Dispositif d'échantillonnage. TPC: terre-plein centrale; $\mathbf{A 0}$ : côté aller juste après le trottoir; A50 : côté aller 50 mètres du trottoir; $\mathbf{A 1 0 0}$ : côté aller 100 mètres du trottoir; $\mathbf{A 1 5 0}$ : côté aller 150 mètres du trottoir; $\mathbf{A 2 0 0}$ : côté aller 200 mètres du trottoir; B0 : côté retour juste après le trottoir; B50 : côté retour 50 mètres du trottoir; B100 : côté retour 100 mètres du trottoir; B150 : côté retour 150 mètres du trottoir; B200 : côté retour 200 mètres du trottoir. 


\section{RESULTATS}

Diversité et distribution des communautés de vers de terre

Au total, 19 espèces de vers de terre ont été collectées. Ces espèces appartiennent principalement à 02 catégories écologiques que sont les épigés et les endogés (Tableau 1). Parmi les vers collectés, les espèces Chuniodrilus palustris, Chuniodrilus zielae, Dichogaster agilis, Eudrilus eugeniae, Hyperiodrilus africanus, Millsonia omodeoi, Millsonia schlegelli et Stuhlmannia prorifera avaient dans l'ensemble de fortes densités et une présence régulière au sein des monolithes réalisés le long du gradient. En revanche, les espèces Agastrodrillus multivesiculatus, Chuniodrilus vuattouxi, Dichogaster arboricola, Dichogaster bolaui, Dichogaster saliens, Dichogaster sp1, Dichogaster sp2, Dichogaster terrae-nigrae, Millsonia ghanaensis et Millsonia lamtoiana avaient de faibles densités et des absences importantes (Tableau 2).

Les paramètres de diversité biologique des vers de terre collectés, notamment, la densité totale, l'indice de diversité de Shannon - Wiever et l'équitabilité ont été très faibles au niveau du terre-plein central $(97,6 \pm 8,67$ ind $/ \mathrm{m}^{2}, 1,48$ et 0,5 , respectivement) alors qu'à $200 \mathrm{~m}$, de part et d'autres de la chaussée, leurs valeurs respectives étaient plus élevées $\left(A_{200}\left(873,6 \pm 56,78 \mathrm{ind} / \mathrm{m}^{2}, 2,32\right.\right.$ et 0,79$)$; $\mathrm{B}_{200}\left(873,6 \pm 56,78 \mathrm{ind} / \mathrm{m}^{2}, 2,55\right.$ et 0,86$\left.)\right)$ (Tableau 2).

Les comparaisons par paire des densités moyennes de vers de terre, réalisées selon le test de Kruskal-Wallis, ont révélé l'existence de différences significatives $(\mathrm{P}<$ $0,05)$ entre les densités moyennes des vers terre, à proximité immédiate de la chaussée (TPC, $\mathrm{A}_{0}, \mathrm{~B}_{0}$ ) et celles les plus éloignées $\left(\mathrm{A}_{100}, \mathrm{~A}_{150}, \mathrm{~A}_{200}, \mathrm{~B}_{100}, \mathrm{~B}_{150}\right.$ et $\left.\mathrm{B}_{200}\right)$ (Tableau 3a et 3b).

Par ailleurs, la classification hiérarchique du nombre d'espèces au sein des différents monolithes réalisés le long du gradient, a été similaire au niveau du terreplein central et des distances $A_{0} ; A_{150}$ et $B_{200}$; $B_{100}$ et $A_{50}$ (Figure 2). Les résultats ont aussi montré que de part et d'autre de l'autoroute, la densité totale des vers de terre et le nombre d'espèces sont plus faibles à proximité immédiate de la chaussée que lorsqu'on s'en éloigne.

\section{Structure des communautés de vers de terre}

La structure des communautés de vers de terre a été mise en évidence par une analyse factorielle des correspondances (AFC). Les résultats de cette AFC ont montré que les 4 premiers axes qui portent l'essentiel des informations sur l'ensemble des facteurs écologiques propres aux monolithes réalisés le long des bordures de l'autoroute et susceptibles d'influencer la distribution des vers ont expliqué à $44,89 \%$, la variance totale de la matrice des vers de terre collectés (Tableau 4). Deux grands groupes d'espèces ont été distingués par l'ordination des communautés de vers de terre dans l'espace réduit formé par les 2 premiers axes, qui ont expliqué, respectivement, $17,49 \%$ et $11,18 \%$ de leur variance totale (Figure 3). Un premier groupe marqué par des espèces fortement corrélées aux axes factoriels était composé des espèces Chuniodrilus palustris, Chuniodrilus zielae, Dichogaster agilis, Millsonia omodeoi, Stuhlmannia prorifera, Agastrodrillus multivesiculatus, Eudrilus eugeniae et Hyperiodrilus africanus. Dans ce groupe, outre l'espèce Dichogaster agilis qui appartient à la catégorie écologique des épigés, toutes les autres espèces étaient des endogées. Un second groupe d'espèces de vers qui se démarquent par leur localisation aux extrémités des axes, était composé des espèces Chuniodrilus vuattouxi, Dichogaster arboricola, Dichogaster bolaui, Dichogaster saliens, Dichogaster sp1, Dichogaster sp2 et Millsonia ghanaensis. Dans ce groupe, hors mis l'espèce Millsonia ghanaensis qui est une espèce endogée, toutes les autres espèces sont des vers épigés. Les espèces, Chuniodrilus vuattouxi et Dichogaster arboricola se retrouvent isolées des autres (Figure 3). 
Interaction entre les communautés de vers de terre et les ETM

Le long du gradient d'échantillonnage, 8 substances ETM ont été détectées et quantifiées, à savoir le cobalt (Co), le chrome $(\mathrm{Cr})$, le cuivre $(\mathrm{Cu})$, le manganèse $(\mathrm{Mn})$, le nickel $(\mathrm{Ni})$, le plomb $(\mathrm{Pb})$, le thallium $(\mathrm{Tl})$ et le zinc $(\mathrm{Zn})$. Leurs concentrations moyennes le long du gradient variaient de $0,007 \pm 0,000$ à $0,295 \pm 0,04 \mathrm{mg} / \mathrm{kg}$ pour le $\mathrm{Co}$; de $0,17 \pm 0.03$ à $5,025 \pm 0,84$ $\mathrm{mg} / \mathrm{kg}$ pour le $\mathrm{Cr}$, de 2,282 $\pm 0,26$ à $20,08 \pm 1,11$ $\mathrm{mg} / \mathrm{kg}$ pour le $\mathrm{Cu}$; de $0,001 \pm 0,000$ à $0,145 \pm 0,03 \mathrm{mg} / \mathrm{kg}$ pour le $\mathrm{Mn}$, de $0,186 \pm 0,051$ à $7,923 \pm 0,941 \mathrm{mg} / \mathrm{kg}$ pour le $\mathrm{Ni}$, de $8,264 \pm 0,553$ à $50,951 \pm 3,133 \mathrm{mg} / \mathrm{kg}$ pour le $\mathrm{Pb}$, de $0,082 \pm 0,008$ à $0,453 \pm 0,02 \mathrm{mg} / \mathrm{kg}$ pour le $\mathrm{Tl}$, de $2,102 \pm 0,275$ à $26,662 \pm 1,47 \mathrm{mg} / \mathrm{kg}$ pour le Zn (Tableau 5).

L'analyse canonique des correspondances (ACC) montre que les 4 premiers axes canoniques qui renseignent le plus sur la relation entre les vers de terre et les ETM ont expliqué $78,57 \%$ de la variance de la matrice des vers de terre collectés (Tableau 6a). Cependant, les 2 premiers axes canoniques qui portent l'essentielle des informations sur la relation étaient présentés par un gradient de thallium, de zinc et de nickel pour l'axe 1 et par celui de plomb et de cuivre pour l'axe 2 (Tableau 6b). Ces deux principaux axes canoniques ont aussi présenté de fortes corrélations avec les espèces Dichogaster bolaui, Dichogaster sp1, Dichogaster sp2, Millsonia ghanaensis (Tableau 6c). Le test de permutation de Monte Carlo a montré que le long des bords de l'autoroute, les ETM ont produit une ordination dans laquelle les premiers axes et tous les autres axes canoniques sont significatives $(\mathrm{p}<0,05)$.

Par ailleurs, selon la comparaison réalisée entre les résultats issus de l'analyse canonique de correspondance (ACC) et les résultats issus de l'analyse factorielle des correspondances (AFC), il est observé que les valeurs propres baissent pour les 2 premiers axes, de 0,401 à 0,272 dans l'analyse de correspondance (AFC), et de 0,036 à 0,024 dans l'analyse canonique de correspondance (ACC) (Tableau 7). Apparemment, les ETM analysés expliquent la principale variance de la matrice des vers de terre extraite par l'AFC.

Tableau 1 : Catégories écologiques des espèces de vers de terre identifiées au niveau des bordures de l'autoroute.

\begin{tabular}{lc}
\hline Espèces de vers de terre & Catégories écologiques \\
\hline Agastrodrillus multivesiculatus & Endogé oligohumique \\
Chuniodrilus palustris & Endogé polyhumique \\
Chuniodrilus vuattouxi & Endogé polyhumique \\
Chuniodrilus zielae & Endogé polyhumique \\
Dichogaster agilis & Epigé détritivore \\
Dichogaster arboricola & Epigé détritivore \\
Dichogaster baeri & Epigé détritivore \\
Dichogaster bolani & Epigé détritivore \\
Dichogaster saliens & Epigé détritivore \\
Dichogaster sp1 & Epigé détritivore \\
Dichogaster sp2 & Epigé détritivore \\
Dichogaster terrae-nigrae & Endogé oligohumique \\
Eudrilus eugeniae & Epigé détritivore \\
Hyperiodrilus africanus & Endogé polyhumique \\
Millsonia ghanaensis & Endogé oligohumique \\
Millsonia lamtoiana & Epigé polyhumique \\
Millsonia omodeoi & Endogé mesohumique \\
Millsonia schlegelli & Endogé polyhumique \\
Stuhlmannia prorifera & Endogé polyhumique \\
\hline
\end{tabular}


M. TOURE et al. / Int. J. Biol. Chem. Sci. 11(5): 2277-2293, 2017

Tableau 2 : Espèces de vers de terre collectées et paramètres de diversité biologique correspondants (densité totale (ind $\left./ \mathrm{m}^{2}\right)$, indice de diversité et équitabilité) aux différents points échantillonnés le long du gradient.

\begin{tabular}{|c|c|c|c|c|c|c|c|c|c|c|c|}
\hline & \multicolumn{5}{|c|}{ Côté retour (B) } & \multirow{2}{*}{$\begin{array}{l}\text { TPC } \\
\text { TPC }\end{array}$} & \multicolumn{5}{|c|}{ Côté aller (A) } \\
\hline & $\mathbf{B 2 0 0}$ & B150 & B100 & B50 & B0 & & A0 & $\mathbf{A 5 0}$ & $\mathbf{A 1 0 0}$ & $\mathbf{A 1 5 0}$ & $\mathbf{A 2 0 0}$ \\
\hline Agastrodrillus multivesiculatus * & $22,4 \pm 3,03$ & $14,4 \pm 2,76$ & $22,4 \pm 5,07$ & $1,6 \pm 0,5$ & $0,00 \pm 0,00$ & $0,00 \pm 0,00$ & $0,00 \pm 0,00$ & $4,8 \pm 1,07$ & $17,6 \pm 2,96$ & $17,6 \pm 1,91$ & $16 \pm 3,45$ \\
\hline Chuniodrilus palustris $* *$ & $233,6 \pm 19,24$ & $189,8 \pm 14,68$ & $118,4 \pm 13,93$ & $28,8 \pm 3,18$ & $59,2 \pm 8,29$ & $9,6 \pm 1,34$ & $19,2 \pm 3,18$ & $43,2 \pm 4,65$ & $56 \pm 5,01$ & $131,2 \pm 14,18$ & $209,6 \pm 19,31$ \\
\hline Chuniodrilus vouattuoxi * & $0,00 \pm 0,00$ & $0,00 \pm 0,00$ & $0,00 \pm 0,00$ & $1,6 \pm 0,5$ & $0,00 \pm 0,00$ & $1,6 \pm 0,5$ & $0,00 \pm 0,00$ & $0,00 \pm 0,00$ & $32 \pm 4,46$ & $12,8 \pm 2,8$ & $52,8 \pm 10,87$ \\
\hline Chuniodrilus zielae ** & $137,6 \pm 12,78$ & $86,4 \pm 6,66$ & $64 \pm 5,05$ & $22,4 \pm 1,87$ & $27,2 \pm 2,82$ & $19,2 \pm 2,9$ & $25,6 \pm 3,38$ & $51,02 \pm 4,25$ & $54,4 \pm 3,78$ & $64 \pm 4,26$ & $137,6 \pm 10,98$ \\
\hline Dichogaster agilis $* *$ & $105,6 \pm 7,73$ & $81,6 \pm 6,72$ & $46,4 \pm 3,57$ & $35,2 \pm 3,52$ & $28,8 \pm 2,8$ & $30,4 \pm 4,02$ & $16 \pm 2,38$ & $19,2 \pm 1,96$ & $100,8 \pm 15,21$ & $64 \pm 6,35$ & $99,2 \pm 8,11$ \\
\hline Dichogaster arboricola $*$ & $6,4 \pm 1,54$ & $4,8 \pm 0,77$ & $0,00 \pm 0,00$ & $1,6 \pm 0,5$ & $3,2 \pm 0,67$ & $0,00 \pm 0,00$ & $0,00 \pm 0,00$ & $0,00 \pm 0,00$ & $12,8 \pm 2,1$ & $6,4 \pm 1,11$ & $33,6 \pm 10,62$ \\
\hline Dichogaster baeri * & $6,4 \pm 2,02$ & $11,2 \pm 1,69$ & $4,8 \pm 1,07$ & $0,00 \pm 0,00$ & $4,8 \pm 0,77$ & $0,00 \pm 0,00$ & $0,00 \pm 0,00$ & $1,6 \pm 0,5$ & $0,00 \pm 0,00$ & $24 \pm 4,96$ & $24 \pm 4,21$ \\
\hline Dichogaster bolaui * & $24 \pm 3,39$ & $4,8 \pm 1,51$ & $9,6 \pm 2,52$ & $4,8 \pm 1,51$ & $0,00 \pm 0,00$ & $0,00 \pm 0,00$ & $0,00 \pm 0,00$ & $1,6 \pm 0,5$ & $4,8 \pm 1,07$ & $3,2 \pm 0,67$ & $0,00 \pm 0,00$ \\
\hline Dichogaster saliens * & $6,4 \pm 1,34$ & $22,4 \pm 5,01$ & $4,8 \pm 1,07$ & $0,00 \pm 0,00$ & $1,6 \pm 0,5$ & $0,00 \pm 0,00$ & $0,00 \pm 0,00$ & $1,6 \pm 0,5$ & $19,2 \pm 5,04$ & $9,6 \pm 1,71$ & $11,2 \pm 2,39$ \\
\hline Dichogaster $\mathrm{sp} 1 *$ & $14,4 \pm 3,05$ & $9,6 \pm 2,15$ & $0,00 \pm 0,00$ & $0,00 \pm 0,00$ & $0,00 \pm 0,00$ & $0,00 \pm 0,00$ & $0,00 \pm 0,00$ & $0,00 \pm 0,00$ & $9,6 \pm 2,02$ & $4,8 \pm 1,51$ & $9,6 \pm 3,03$ \\
\hline Dichogaster $\mathrm{sp} 2 *$ & $16 \pm 5,05$ & $11,2 \pm 3,54$ & $3,2 \pm 0,67$ & $0,00 \pm 0,00$ & $0,00 \pm 0,00$ & $0,00 \pm 0,00$ & $0,00 \pm 0,00$ & $1,6 \pm 0,5$ & $1,6 \pm 0,5$ & $12,8 \pm 2,59$ & $0,00 \pm 0,00$ \\
\hline Dichogasterterae-nigrae * & $11,2 \pm 2,13$ & $11,2 \pm 1,51$ & $11,2 \pm 2$ & $9,6 \pm 1,71$ & $3,2 \pm 1,01$ & $0,00 \pm 0,00$ & $0,00 \pm 0,00$ & $0,00 \pm 0,00$ & $12,9 \pm 2,13$ & $14,4 \pm 3,57$ & $8 \pm 1,35$ \\
\hline Eudrilus engeniae $* *$ & $4,8 \pm 1,07$ & $14,4 \pm 2,31$ & $4,8 \pm 1,07$ & $0,00 \pm 0,00$ & $1,6 \pm 0,5$ & $1,6 \pm 0,5$ & $3,2 \pm 1,01$ & $1,6 \pm 0,5$ & $1,6 \pm 0,5$ & $1,6 \pm 0,5$ & $3,2 \pm 1,01$ \\
\hline Hyperiodrilus africanus $* *$ & $22,4 \pm 2,28$ & $24 \pm 1,88$ & $8 \pm 1,35$ & $3,2 \pm 0,67$ & $3,2 \pm 0,67$ & $4,8 \pm 1,07$ & $0,00 \pm 0,00$ & $3,2 \pm 0,67$ & $9,6 \pm 1,71$ & $17,6 \pm 2,05$ & $14,4 \pm 2,55$ \\
\hline Millsonia ghaneansis * & $8 \pm 1,72$ & $0,00 \pm 0,00$ & $1,6 \pm 0,5$ & $0,00 \pm 0,00$ & $0,00 \pm 0,00$ & $0,00 \pm 0,00$ & $0,00 \pm 0,00$ & $0,00 \pm 0,00$ & $0,00 \pm 0,00$ & $9,6 \pm 2,15$ & $0,00 \pm 0,00$ \\
\hline Millsonia lamtoiana * & $22,4 \pm 2,52$ & $14,4 \pm 1,91$ & $14,4 \pm 2,05$ & $6,4 \pm 1,11$ & $4,8 \pm 0,77$ & $0,00 \pm 0,00$ & $0,00 \pm 0,00$ & $3,2 \pm 1,011$ & $16 \pm 1,84$ & $22,4 \pm 2,4$ & $32 \pm 1,84$ \\
\hline Millsonia omedeoi $* *$ & $64 \pm 6,48$ & $43,2 \pm 2,72$ & $36,8 \pm 4,13$ & $20,8 \pm 2,72$ & $14,4 \pm 1,91$ & $11,2 \pm 1,07$ & $4,8 \pm 1,51$ & $24 \pm 2,29$ & $65,6 \pm 3,95$ & $83,2 \pm 4,18$ & $115,2 \pm 7,42$ \\
\hline Millsonia schelegilli ** & $16 \pm 1,84$ & $30,4 \pm 3,87$ & $17,6 \pm 2,05$ & $4,8 \pm 1,07$ & $3,2 \pm 0,67$ & $1,6 \pm 0,5$ & $1,6 \pm 0,5$ & $16 \pm 3,69$ & $27,2 \pm 4,13$ & $27,2 \pm 2,92$ & $48 \pm 7,54$ \\
\hline Stuhlmannia prorifera $* *$ & $24 \pm 4,41$ & $28,8 \pm 2,9$ & $19,2 \pm 2,23$ & $8 \pm 0,84$ & $4,8 \pm 0,77$ & $17,6 \pm 1,76$ & $6,4 \pm 1,54$ & $38,4 \pm 6,09$ & $25,6 \pm 3,12$ & $84,8 \pm 7,84$ & $59,2 \pm 3,29$ \\
\hline Densité totale (ind/m²) & $745,6 \pm 59,21$ & $602,6 \pm 45,23$ & $387,2 \pm 29,11$ & $148,8 \pm 10,7$ & $160 \pm 14,94$ & $97,6 \pm 8,67$ & $76,8 \pm 7,63$ & $211,02 \pm 16,41$ & $467,3 \pm 26,86$ & $611,2 \pm 35,82$ & $873,6 \pm 56,78$ \\
\hline Indice de diversité de Shannon - & & & & & & & & & & & \\
\hline Wiener & 2,55 & 2,53 & 2,43 & 2,13 & 2,11 & 1,48 & 1,63 & 2,1 & 2,48 & 2,55 & 2,32 \\
\hline Equitabilité & 0,86 & 0,87 & 0,83 & 0,72 & 0,72 & 0,5 & 0,55 & 0,71 & 0,84 & 0,87 & 0,79 \\
\hline
\end{tabular}
** : espèces de vers à fortes densités et une présence régulière; * : espèces de vers à faibles densités et des absences importantes; TPC: terre-plein centrale; $\mathbf{A 0}:$ côté aller juste après le trottoir; A50 : côté
aller 50 mètres du trottoir; $\mathbf{A 1 0 0}:$ côté aller 100 mètres du trottoir; $\mathbf{A 1 5 0}:$ côté aller 150 mètres du trottoir; $\mathbf{A 2 0 0}:$ côté aller 200 mètres du trottoir; $\mathbf{B 0}:$ côté retour juste après le trottoir; B50 : côté retour 50 mètres du trottoir; B100: côté retour 100 mètres du trottoir; B150: côté retour 150 mètres du trottoir; B200 : côté retour 200 mètres du trottoir. 
Tableau 3a : Résultats du test de Kruskal-Wallis (valeurs p) pour la distribution des densités de vers de terre du côté aller (A) de l'autoroute (Abidjan-Singrobo).

\begin{tabular}{|c|c|c|c|c|c|c|}
\hline & \multicolumn{6}{|c|}{ Côté aller (A) } \\
\hline & TPC & A 0 & A 50 & A 100 & A 150 & A 200 \\
\hline TPC & - & & & & & \\
\hline A 0 & 1,000000 & - & & & & \\
\hline A 50 & 1,000000 & 1,000000 & - & & & \\
\hline A 100 & $0,028631 * *$ & $0,009578 * *$ & 1,000000 & - & & \\
\hline A 150 & $0,001857 * *$ & $0,000518 * *$ & 0,329862 & 1,000000 & _ & \\
\hline A 200 & $0,000030 * *$ & $0,000007 * *$ & $0,016605 * *$ & 1,000000 & 1,000000 & 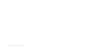 \\
\hline
\end{tabular}

Tableau 3b : Résultats du test de Kruskal-Wallis (valeurs p) pour la distribution des densités de vers de terre du côté retour (B) de l'autoroute (Singrobo-Abidjan).

\begin{tabular}{|c|c|c|c|c|c|c|}
\hline & \multicolumn{6}{|c|}{ Côté retour (B) } \\
\hline & TPC & В 0 & B 50 & B 100 & B 150 & B 200 \\
\hline TPC & _ & & & & & \\
\hline В 0 & 1,000000 & - & & & & \\
\hline B 50 & 1,000000 & 1,000000 & - & & & \\
\hline B 100 & 0,178086 & 1,000000 & 1,000000 & _ & & \\
\hline B 150 & $0,001801 * *$ & $0,040552 * *$ & $0,031769 * *$ & 1,000000 & _ & \\
\hline B 200 & $0,000440 * *$ & $0,012468 * *$ & $0,009578 * *$ & 1,000000 & 1,000000 & \\
\hline
\end{tabular}

Tableau 4 : Résultats de l'analyse factorielle des correspondances de la communauté des vers de terre.

\begin{tabular}{lrrrr}
\hline & Ax1 & Ax2 & Ax3 & Ax4 \\
\hline Valeurs propres & 0.401 & 0.272 & 0.181 & 0.174 \\
Pourcentage de la variance des vers de terre & 17.487 & 11.871 & 7.917 & 7.617 \\
Pourcentage cumulé de la variance des vers de terre & 17.49 & 29.36 & 37.28 & $\mathbf{4 4 . 8 9}$ \\
Inertie totale & & 2.291 & & \\
\hline
\end{tabular}


M. TOURE et al. / Int. J. Biol. Chem. Sci. 11(5): 2277-2293, 2017

Tableau 5 : Concentrations moyennes $(\mathrm{mg} / \mathrm{kg})$ des substances ETM détectées et quantifiées le long du gradient d'échantillonnage.

\begin{tabular}{|c|c|c|c|c|c|c|c|c|c|c|c|}
\hline & \multicolumn{5}{|c|}{ Côté retour (B) } & \multicolumn{2}{|l|}{ TPC } & \multicolumn{3}{|c|}{ Côté aller (A) } & \multirow[b]{2}{*}{$\mathbf{A 2 0 0}$} \\
\hline & B200 & B150 & B100 & B50 & B0 & TPC & A0 & A50 & $\mathbf{A 1 0 0}$ & A150 & \\
\hline Cobalt & $0,007 \pm 0,000$ & $0,022 \pm 0,003$ & $0,034 \pm 0,002$ & $0,07 \pm 0,005$ & $0,101 \pm 0,01$ & $0,295 \pm 0,04$ & $0,101 \pm 0,01$ & $0,061 \pm 0,004$ & $0,027 \pm 0,00$ & $0,016 \pm 0,001$ & $0,043 \pm 0.006$ \\
\hline Chrome & $0,449 \pm 0,09$ & $1,156 \pm 0,14$ & $1,84 \pm 0,38$ & $2,723 \pm 0,45$ & $5,025 \pm 0,84$ & $2,921 \pm 0,22$ & $1,16 \pm 0,12$ & $0,963 \pm 0,07$ & $0,37 \pm 0,03$ & $0,177 \pm 0,03$ & $0,00 \pm 0,00$ \\
\hline Cuivre & $2,282 \pm 0,26$ & $4,197 \pm 0,52$ & $6,17 \pm 0,49$ & $8,765 \pm 0,75$ & $14,643 \pm 0,95$ & $20,03 \pm 1,11$ & $19,258 \pm 0,97$ & $11,229 \pm 0,69$ & $6,93 \pm 0,26$ & $4,903 \pm 0,41$ & $2,51 \pm 0,29$ \\
\hline Manganèse & $0,002 \pm 0,000$ & $0,005 \pm 0,001$ & $0,012 \pm 0,001$ & $0,013 \pm 0,001$ & $0,024 \pm 0,002$ & $0,145 \pm 0,03$ & $0,02 \pm 0,001$ & $0,013 \pm 0,001$ & $0,009 \pm 0,001$ & $0,005 \pm 0,001$ & $0,001 \pm 0,000$ \\
\hline Nickel & $0,186 \pm 0,051$ & $0,451 \pm 0,083$ & $0,638 \pm 0,092$ & $0,943 \pm 0,116$ & $1,187 \pm 0,16$ & $7,923 \pm 0,941$ & $2,064 \pm 0,082$ & $1,744 \pm 0,163$ & $1,063 \pm 0,061$ & $0,552 \pm 0,061$ & $0,268 \pm 0,047$ \\
\hline Plomb & $14,482 \pm 1,161$ & $21,952 \pm 1,743$ & $25,729 \pm 1,6$ & $31,431 \pm 1,52$ & $50,951 \pm 3,133$ & $48,74 \pm 1,091$ & $49,309 \pm 0,664$ & $36,124 \pm 1,322$ & $29,364 \pm 0,851$ & $19,715 \pm 0,841$ & $8,264 \pm 0,553$ \\
\hline Thallium & $0,082 \pm 0,008$ & $0,137 \pm 0,009$ & $0,166 \pm 0,01$ & $0,268 \pm 0,007$ & $0,374 \pm 0,01$ & $0,315 \pm 0,03$ & $0,453 \pm 0,02$ & $0,285 \pm 0,012$ & $0,229 \pm 0,01$ & $0,168 \pm 0,01$ & $0,095 \pm 0,005$ \\
\hline Zinc & $3,195 \pm 0,391$ & $5,969 \pm 0,58$ & $7,638 \pm 0,581$ & $19,041 \pm 2,072$ & $28 \pm 3,19$ & $26,662 \pm 1,47$ & $24,234 \pm 1,593$ & $17,996 \pm 1,071$ & $12,041 \pm 0,842$ & $7,942 \pm 0,67$ & $2,102 \pm 0,275$ \\
\hline
\end{tabular}

TPC: terre-plein centrale; $\mathbf{A 0}:$ côté aller juste après le trottoir; $\mathbf{A 5 0}:$ côté aller 50 mètres du trottoir; $\mathbf{A 1 0 0}$ : côté aller 100 mètres du trottoir; $\mathbf{A 1 5 0}:$ côté aller 150 mètres du trottoir; $\mathbf{A 2 0 0}:$ côté aller 200 mètres du trottoir; B0 : côté retour juste après le trottoir; B50 : côté retour 50 mètres du trottoir; B100 : côté retour 100 mètres du trottoir; B150 : côté retour 150 mètres du trottoir; B200 : côté retour 200 mètres du trottoir. 
Tableau 6a : Résultats de l'analyse canonique des correspondances.

\begin{tabular}{lrrrr}
\hline & Ax1 & Ax2 & Ax3 & Ax4 \\
\hline Valeurs propres & 0,036 & 0,024 & 0,017 & 0,013 \\
Pourcentage de la variance des vers de terre et des ETM & 31,76 & 20,63 & 14,49 & 11,69 \\
Pourcentage cumulé de la variance des vers de terre et des ETM & 31,76 & 52,39 & 66,88 & $\mathbf{7 8 , 5 7}$ \\
Inertie totale & \multicolumn{2}{c}{0,115} \\
\hline
\end{tabular}

Tableau 6b : Coefficients canoniques des ETM.

\begin{tabular}{ccccccccc}
\hline & Cobalt & Chrome & Cuivre & Manganèse & Nickel & Plomb & Thallium & Zinc \\
\hline Axe 1 & 0,417 & 0,116 & 0,389 & 0,359 & $\mathbf{0 , 5 2 6}$ & 0,001 & $\mathbf{0 , 7 7 9}$ & $\mathbf{0 , 5 4 8}$ \\
Axe 2 & 0,341 & 0,004 & $\mathbf{0 , 5 7 6}$ & 0,162 & 0,070 & $\mathbf{0 , 8 6 4}$ & 0,366 & 0,060 \\
\hline
\end{tabular}

Tableau 6c : Corrélation entre les vers de terre et les axes canoniques.

\begin{tabular}{lcc}
\hline Espèces & Axe 1 & Axe 2 \\
\hline Agastrodrillus multivesiculatus & 0,069 & 0,090 \\
Chuniodrilus palustris & 0,198 & $-0,124$ \\
Chuniodrilus vouattuoxi & $-0,267$ & 0,239 \\
Chuniodrilus zielae & $-0,031$ & $-0,035$ \\
Dichogaster agilis & 0,237 & $-0,019$ \\
Dichogaster arboricola & 0,163 & 0,093 \\
Dichogaster baeri & 0,216 & 0,327 \\
Dichogaster bolani & $-0,250$ & $\mathbf{0 , 5 2 5}$ \\
Dichogaster saliens & 0,295 & $-0,033$ \\
Dichogaster sp1 & $\mathbf{0 , 5 0 1}$ & 0,362 \\
Dichogaster sp2 & 0,324 & $\mathbf{0 , 5 2 7}$ \\
Dichogaster terrae-nigrae & $-0,003$ & $-0,299$ \\
Eudrilus eugeniae & 0,037 & 0,393 \\
Hyperiodrilus africanus & $-0,291$ & 0,135 \\
Millsonia ghanaensis & 0,168 & $\mathbf{0 , 7 3 2}$ \\
Millsonia lamtoiana & 0,019 & 0,178 \\
Millsonia omodeoi & 0,045 & 0,066 \\
Millsonia schlegelli & 0,044 & 0,054 \\
Stuhlmannia prorifera & $-0,304$ & $-0,136$ \\
\hline
\end{tabular}


Tableau 7 : Comparaison de l'analyse canonique des correspondances et de l'analyse factorielle des correspondances.

\begin{tabular}{lcccc}
\hline & Axe 1 & Axe 2 & Axe 3 & Axe 4 \\
\hline AFC & & & & \\
Valeurs propres & 0,401 & 0,272 & 0,181 & 0,175 \\
pourcentage de la variance des vers de terre & 17,487 & 11,871 & 7,917 & 7,617 \\
pourcentage cumulé de la variance des vers de terre & 17,49 & 29,36 & 37,28 & 44,89 \\
ACC & & & & \\
$\begin{array}{l}\text { Valeurs propres } \\
\begin{array}{l}\text { Pourcentage de la variance des vers de terre et des } \\
\text { substances polluantes }\end{array}\end{array}$ & 0,036 & 0,024 & 0,017 & 0,013 \\
$\begin{array}{l}\text { Pourcentage cumulé de la variance des vers de terre } \\
\text { et des substances polluantes }\end{array}$ & 31,76 & 20,63 & 14,49 & 11,69 \\
\hline
\end{tabular}

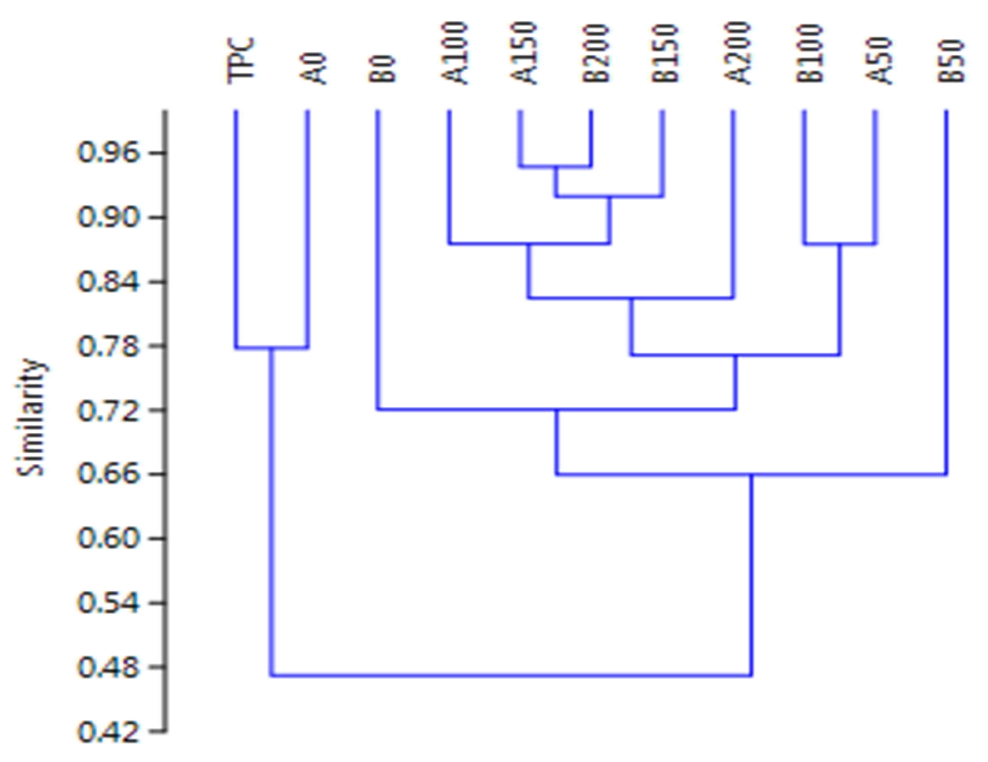

Figure 2 : Dendrogramme de la classification des indices de similarités de Jacquard. TPC: terre-plein centrale; A0 : côté aller juste après le trottoir; A50 : côté aller 50 mètres du trottoir; $\mathbf{A 1 0 0}$ : côté aller 100 mètres du trottoir; A150 : côté aller 150 mètres du trottoir; $\mathbf{A 2 0 0}$ : côté aller 200 mètres du trottoir; $\mathbf{B 0}$ : côté retour juste après le trottoir; B50 : côté retour 50 mètres du trottoir; B100 : côté retour 100 mètres du trottoir; B150 : côté retour 150 mètres du trottoir; B200 : côté retour 200 mètres du trottoir. 


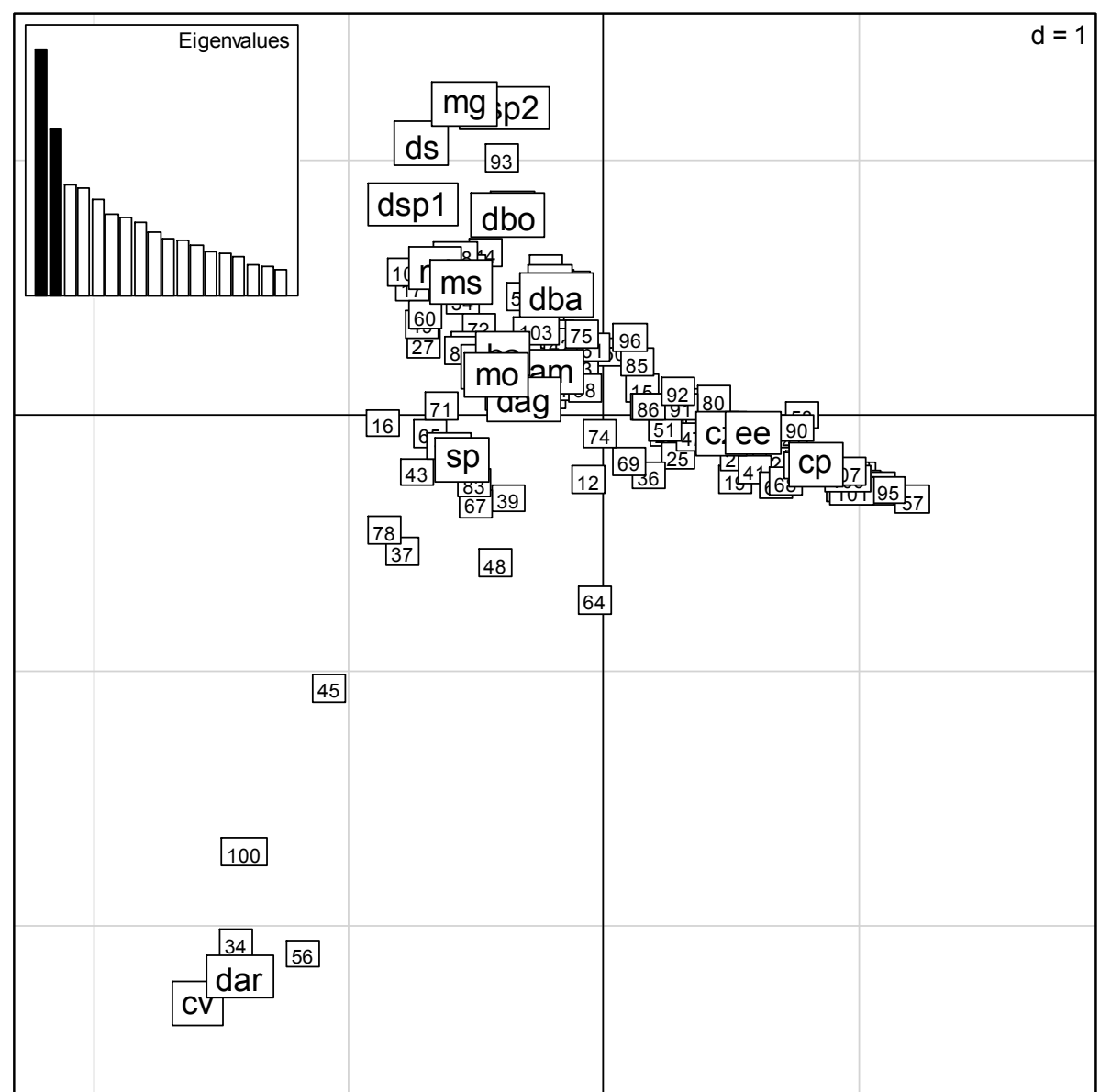

Figure 3 : Analyse factorielle de correspondance des communautés de vers terre. am : Agastrodrillus multivesiculatus; cp : Chuniodrilus palustris; cv : Chuniodrilus vouattuoxi; cz: Chuniodrilus zielae; dag : Dichogaster agilis ; dar : Dichogaster arboricola ; dba : Dichogaster baeri ; dbo : Dichogaster bolani ; ds : Dichogaster saliens ; dsp1 : Dichogaster sp1; dsp2 : Dichogaster sp2; dtn : Dichogaster terrae-nirae; ee : Eudrilus engeniae; ha : Hyperiodrilus africanus ; mg : Millsonia ghaneensis ; ml : Millsonia lamtoiana ; mo : Millsonia omodeoi ; ms : Millsonia schelegilli ; sp : Stuhlmannia prorifera.

\section{DISCUSSION}

Distribution et structuration des communautés de vers de terre

La densité des vers de terre collectés et le nombre d'espèces ont été faibles à proximité immédiate de la chaussée. Par contre, ces valeurs deviennent de plus en plus élevées lorsqu'on s'en éloigne. Ce gradient de distribution des densités et des espèces de vers en bordure de l'autoroute résulterait des contraintes du milieu, notamment, la présence des substances polluantes et les perturbations dues au trafic routier. En effet, les bords de routes et d'autoroutes sont considérés comme des réservoirs importants de substances polluantes (Durand, 2003). Lévêque et al. (2015) ont révélé que la présence des ETM dans les sols, influence significativement la survie des vers de terre. Par ailleurs, selon Rossi (2003), la modification de la répartition des vers de terre dans un milieu serait due à l'hétérogénéité spatiale comme une réponse aux facteurs abiotiques, aux comportements grégaires ou au mode de reproduction. L'ordination des espèces de vers de terre par l'analyse factorielle des correspondances (AFC) a permis de mesurer la principale variabilité de la communauté (environ $45 \%$ pour les 4 premiers axes) le long des bordures de l'autoroute. Par conséquent, la distribution 
de ces organismes serait en grande partie liée à la présence des substances polluantes ou un probable processus stochastique lié aux erreurs d'échantillonnage. En effet, l'interprétation du pourcentage de variance mesurée tient-compte du fait que le pourcentage n'atteint jamais $100 \%$, car une partie de cette variance peut être due aux "bruits" des données qui sont interprétés comme étant la résultante d'une fluctuation aléatoire des espèces (Ter Braak et Šmilauer, 2002). La structure des données est résumée, selon cet auteur, dans les premiers axes principaux tandis que les autres seraient expliqués par le bruit des données. Une ordination qui explique seulement un faible pourcentage peut néanmoins contenir une bonne partie de l'information écologique. Dans le plan factoriel formé par les deux premiers axes, le groupe caractérisé par les espèces ayant une densité élevée et une distribution fréquente serait constitué par les espèces communes des bordures de l'autoroute. Elles sont en majeur partie composées d'espèces endogées qui se nourrissent de sol mélangés à la matière organique. Ces vers sont plus exposés à la pollution (Blouin et al., 2013). L'abondance élevée et la bonne répartition de ces espèces endogées dans ce milieu pourraient se justifier par une excellente capacité d'adaptation. Selon Rossi (200 3) et Pelosi et al. (2014), cette adaptation pourrait être un changement de comportement en réponse aux contraintes $\mathrm{du}$ milieu, telles la pollution et la recherche de la nourriture. Ainsi, certains vers de terre filiformes appartenant à la famille des Eudrilidae sont inféodés aux racines, puis d'autres suivent le gradient d'infiltration de l'eau dans les couches profondes du sol en filtrant les particules organiques (Blouin et al., 2013). Le second groupe caractérisé par des espèces à faibles densités et peu fréquentes, serait constitué par les espèces rares de ces bords d'autoroute. Ces espèces sont en majeur partie composées des vers épigées, qui vivent à la surface du sol dont les faibles abondances et répartitions pourraient se justifier par le fait qu'ils soient directement exposés aux contraintes du milieu. Selon Blouin et al. (2013), ces espèces seraient sensibles à la prédation, aux variations climatiques et aux facteurs anthropiques. De plus, Pilosi et al. (2008) a montré que ces espèces sont assez rares en milieu fortement perturbé. En somme, ces résultats révèlent que la distribution et la structure de la communauté des vers de terre collectés le long des bordures de l'autoroute seraient fonction de la distribution des substances polluantes (ETM) détectées.

\section{Corrélation entre les communautés de vers et les substances ETM}

D’une façon générale, les techniques d'ordination supposent l'existence d'une structure latente dans les données, c'est-à-dire que la répartition des espèces de vers de terre (variable expliquée) au niveau des bordures de l'autoroute serait déterminée par quelques variables environnementales (variables latentes ou variables explicatives) selon la réponse du modèle. Dans la présente étude nous avons supposé que les ETM étaient ces variables explicatives. Ainsi, les ordinations canoniques combinent deux aspects : l'ordination ordinaire et la régression. Les valeurs propres obtenues dans l'ACC sont toujours plus petites que celles obtenues dans l'AFC, à cause de la restriction imposée par les scores du site à être une combinaison linéaire des variables explicatives (Ter Braak et Šmilauer, 2002; Cibois, 2014). La comparaison entre les valeurs propres de l'AFC et celles de l'ACC montre que l'ampleur de la variance expliquée par les substances polluantes pourrait interférer sur la prédiction de la variance principale dans la combinaison des espèces de vers de terre extraite par l'AFC (la variance expliquée par les substances polluantes dans ACC est $78,57 \%$ des $44,89 \%$ de la variance des vers de terre extraite par AFC). Ceci exprimerait que la distribution des vers de terre le long des bords de cette autoroute serait principalement expliquée par les ETM (Co, Cr, Cu, Mn, Ni, $\mathrm{Pb}, \mathrm{Tl}$ et $\mathrm{Zn}$ ) identifiés. L'ACC montre également de fortes corrélations entre les concentrations de thallium, de zinc, de nickel, 
de plomb et de cuivre et les 2 principaux axes canoniques. Ce qui indique que ces substances seraient les principaux polluants influençant la survie des vers de terre au niveau des bordures de l'autoroute. En effet, bien qu'étant indispensables, à l'état de trace pour de nombreux processus cellulaires chez les vers de terre, le cuivre, le nickel et le zinc peuvent se révéler toxiques à forte concentration, et avoir des effets néfastes tels que la réduction de leur biomasse et la réduction de la production de cocon (Scott-Fordsmand et al., 2000 ; Maboeta et al., 2004). Il en est de même pour le plomb (Spurgeon et al., 2003 ; Langdon et al., 2005) et le thallium (Karbowska, 2016), qui ne jouent aucun rôle dans les processus cellulaires des vers de terre, mais s'avèrent plutôt toxiques, même à de faibles concentrations, pour les organismes biologiques. Par ailleurs, les fortes corrélations observées entre les espèces de vers Dichogaster bolaui, Dichogaster sp1, Dichogaster sp2 et Millsonia ghanaensis et les principaux axes canoniques montrent que ces vers seraient les plus impactés par les substances ETM détectées le long des bordures de l'autoroute. Ces résultats ont montré que les substances polluantes, notamment, les ETM ont une influence sur les communautés de vers de terre des bordures de l'autoroute du nord, en Côte d'Ivoire. Ces substances pourraient avoir un impact négatif considérable sur la distribution et la survie de ces organismes.

\section{Conclusion}

L'analyse de la distribution et la structuration des communautés de vers de terre montre que de part et d'autres de l'autoroute du nord, en Côte d'Ivoire, la distribution des vers de terre est caractérisée par une augmentation des densités totales et $\mathrm{du}$ nombre d'espèces au fur et à mesure qu'on s'éloigne de la chaussée. Leur structuration est marquée par une prépondérance des espèces endogées qui seraient les espèces communes et une faible présence des espèces épigées qui seraient les espèces rares. Par ailleurs, l'analyse de la corrélation entre les communautés de vers de terre et les substances polluantes (ETM) le long des bordures de cette autoroute montre que les ETM ( $\mathrm{Co}, \mathrm{Cr}, \mathrm{Cu}, \mathrm{Mn}, \mathrm{Ni}, \mathrm{Pb}, \mathrm{Tl}$ et $\mathrm{Zn}$ ), expliquent principalement les variabilités des communautés de vers de terre. Le thallium, le zinc, le nickel, le plomb et le cuivre sont les principaux polluants qui influencent la survie des communautés des vers de terre le long des bordures de l'autoroute. Cependant, l'impact des ETM est plus fort sur les espèces de vers Dichogaster bolani, Dichogaster sp1, Dichogaster sp2 et Millsonia ghanaensis.

\section{CONFLIT D'INTERETS}

Les auteurs déclarent qu'il n'y a aucun conflit d'intérêts.

\section{CONTRIBUTIONS DES AUTEURS}

TM a supervisé l'ensemble du travail, a contribué à la collecte des données et à la rédaction du manuscrit. TS a participé à l'élaboration du protocole d'échantillonnage, a supervisé le travail, a analysé les données et contribué à la rédaction du manuscrit. ENM a contribué à l'identification des vers des de terre, a analysé les données et contribué à la rédaction du manuscrit. KKKG a participé à la collecte des données, a analysé les données et contribué à la rédaction du manuscrit.

\section{REMERCIEMENTS}

Nos remerciements vont à l'endroit du Laboratoire du Département d'Analyse et de Recherche de la Société Nationale d'Opération Pétrolière (PETROCI) et du Laboratoire National d'Appui au Développement Agricole (LANADA). Merci également aux Professeurs Kolo YEO et Souleymane KONATE, respectivement Responsable de la Station de Recherche en Ecologie de Lamto et Directeur de l'Unité de Recherche en Ecologie et Biodiversité de l'Université Nangui Abrogoua, pour le soutien logistique dans la réalisation de cette étude.

\section{REFFERENCES}

Baby J, Raj JS, Biby ET, Sankarganesh P, Jeevitha MV, Ajisha SU, Rajan SS. 2010. Toxic effect of heavy metals on 
aquatic environment. Int. J. Biol. Chem. Sci., 4(4): 939-952. DOI : http://dx.doi.org/10.4314/ijbcs.v4i4.6297 6.

Banque Mondiale. 2003. Initiative sur la qualité de l'air dans les villes d'Afrique sub-saharienne. Rapport d'avancement 1998-2002. Document de travail numéro 11. http://www.worldbank.org/afr/ssatp. Référence du 13-03-2007.

Bentum JK, Essumang DK, Tuffuor JK, Agyakum I. 2010. Analysis of heavy metals in roadside soils and crops along the Obansandjo way in the Accra metropolis. Int. J. Biol. Chem. Sci., 4(3): 803-808. DOI: http://dx.doi.org/ 10.4314/ijbcs.v4i3.60517.

Blouin M, Hodson ME, Delgado EA, Baker G, Brussaard L, Butt KR, Dai J, Dendooven L, Peres G, Tondoh JE, Cluzeau D, Brun JJ. 2013. A review of earthworm impact on soil function and ecosystem services. Eur. J. Soil Sci., 64: 161-182. DOI: http://dx.doi.org/10.1111/ ejss. 12025 .

Brown GG, Barois I, Lavelle P. 2000. Regulation of soil organic matter dynamics and microbial activity in the drilosphere and the role of interactions with other edaphic functional domains. Eur. J. Soil Biol., 36: 177-198. DOI: http://dx.doi.org/10.1016/S11645563(00)01062-1.

Cibois P. 2014. L'analyse factorielle des correspondances In Les Méthodes d'Analyse d'Enquête. ENS Editions: Lyon; DOI: http://dx.doi.org/10.4000/books.enseditio ns. 1460 .

Conder JM, Lanno RP, Basta NT. 2001. Assessment of metal availability in smelter soil using earthworms and chemical extractions. J. Environemental Qual., 30 : 1231-1237. DOI: http://dx.doi.org/10.2134/jeq2001.30412 $31 \mathrm{x}$.

Csuzdi C, Tondoh JE. 2007. New and littleknown earthworm species from the Ivory Coast (Oligochaeta: Acanthodrilidae: Benhamiinae and Eudrilidae). J. Nat. Hist., 41: 2551-2567. DOI: http://dx.doi.org/10.1080/002229307017 42629.

Durand C. 2003. Caractérisation physicochimique des produits de l'assainissement pluvial. Origine et devenir des métaux traces et des polluants organiques. $\mathrm{PhD}$ Thesis, Poitiers University, France, p. 248.

FER (Fond d'Entretien Routier). 2016. Rapport interne $\mathrm{du}$ fond d'entretien routier Côte-d'Ivoire. http://fer-ci.org/.

Hale CM, Frelich LE, Reich PB. 2005. Exotic European earthworm invasion dynamics in northern hardwood forests of Minnesota, USA. Ecol. Appl., 15:848860. DOI : http://www.jstor.org/stable/ 4543400.

Huynh TMD. 2009. Impact des métaux lourds sur les interactions plante/ ver de terre/ microflore tellurique. Thèse de Doctorat, Université Paris-Est, Paris, p. 170.

Jackson MM. 2009. Assessment of air pollution in residential areas of Kinondoni municipality in Dar es Salaam City, Tanzania. Int. J. Biol. Chem. Sci., 3(4): 786-795. DOI: http://dx.doi.org/10.4314/ijbcs.v3i4.4716 4.

Jolly YN, Ashraful I, Shawkat A. 2013. Transfer of metals from soil to vegetables and possible health risk assessment. SpringerPlus, 2(1): 385-392. DOI: http://dx.doi.org/10.1186/21931801-2-385.

Karbowska B. 2016. Presence of thallium in the environment: sources of contaminations, distribution and monitoring methods. Envi. 
Monit. Assess., 188(11): 640. DOI: http://dx.doi.org/10.1007/s10661-0165647-y.

Kihampa C, Mwegoha WJS. 2010. Heavy metals accumulation in vegetables grown along the Msimbazi River in Dar es Salaam, Tanzania. Int. J. Biol. Chem. Sci., 4(6): 1932-1938. DOI: http://dx.doi.org/10.4314/ijbcs.v4i6.6494 7.

Langdon CL, Hodson ME, Arbold RE, Black S. 2005. Survival, Pb-uptake and behaviour of three species of earthworm in $\mathrm{Pb}$ treated soils determined using an OECD-style toxicity test and a soil avoidance test. Environ. Pollut., 138: 368-375. DOI: https://doi.org/10.1016/ j.envpol.2005.03.002.

Lavelle P. 1978. Les vers de terre de la savane de Lamto (Côte d'Ivoire): peuplements, populations et fonctions dans l'écosystème. Doctorat thèse, Université Paris VI, Paris, p. 301.

Lavelle P, Decaëns T, Aubert M, Barot S, Blouin M, Bureau F, Margerie P, Mora P, Rossi JP. 2006. Soil invertebrates and ecosystem services. Eur. J. Soil Biol., 42: S3-S15. DOI : http://dx.doi.org/ 10.1016/j.ejsobi.2006.10.002.

Lebreton JD, Chessel D, Richardot-Coolet M, Yoccoz N. 1988. L'analyse des relations espèces - milieu par l'analyse canonique des correspondances. I. variable de milieux quantitatifs. Acta Oecologia Oecology Generalis, 9(2): 137-151. 0243-766X/88/02/137/15/\$ 1350/C Gauthier-Villars.

Lévêque T, Capowiez Y, Schreck E, Mombo S, Mazzia C, Foucault Y, Dumat C. 2015. Effects of historic metal (loid) pollution on earthworm communities. Sci. Total Environ., 511: 738-746. DOI: http://dx.doi.org/10.1016/j.scitotenv.201 4.11.101.
Maboeta MS, Reinecke SA, Reinecke AJ. 2004. The relationship between lysosomal biomarker and organismal responses in an acute toxicity test with Eisenia fetida (Oligochaeta) exposed to the fungicide copper oxychloride. Environ. Res., 96: 95-101. DOI : http://dx.doi.org/10.1016/S00139351(03)00138-5.

N'Guessan HJK. 2010. L'invasion des véhicules d'occasion en transit par le port d'Abidjan: le dynamisme ambivalent d'une activité en plein essor. Les Cahiers d'Outre-Mer, 251: 365-390. DOI : http://dx.doi.org/ 10.4000/com.6044.

Pelosi C, Bertrand M, Makowski D, RogerEstrade J. 2008. WORMDYN: A model of Lumbricus terrestris population dynamics in agricultural fields. Ecol. Model., 218(3-4): 219-234. DOI: http://dx.doi.org/10.1016/j.ecolmodel.20 08.07.002.

Pelosi C, Barot S, Capowiez Y, Hedde M, Vandenbulcke F. 2014. Pesticides and earthworms. A review. Agron. Sustain. Dev., 34(1): 199-228. http://dx.doi.org/ 10.1007/s13593-013-0151-z.

Public Eye investigation. 2016. DIRTY DIESEL - How Swiss Traders Flood Africa with Toxic Fuels. www.publiceye.ch, référence du 15 septembre 2016.

Rossi JP. 2003. The spatio-temporal pattern of tropical earthworm species assemblage and its relationship with soil structure: The 7th international symposium on earthworm ecology - Cardiff - Wales · 2002, Pedobiologia, 47: 497-503. DOI: http://dx.doi.org/10.1078/0031-405600219.

Scott-Fordsmand JJ, Weeks JM, Hopkin SP. 2000. Importance of contamination history for understanding toxicity of copper to earthworm Eisenia 
fetica (Oligochaeta: Annelida), using neutral-red retention assay. Environ. Tox. Chem., 19: 1774-1780. DOI: http://dx.doi.org/10.1002/etc.562019071 0 .

Singh A, Rajesh KS, Madhoolika A, Fiona MM. 2010. Health risk assessment of heavy metals via dietary intake of foodstuffs from the wastewater irrigated site of a dry tropical area of India. Food Chem. Toxicol., 48: 611-619. DOI: http://dx.doi.org/10.1016/j.fct.2009.11.0 41.

Spurgeon DJ, Weels JM, Van Gestel CAM. 2003. A summary of eleven years progress in earthworm ecotoxicology. Pedobiologia, 47: 558-606. DOI: https://doi.org/10.1078/0031-405600234.

Ter Braak CJF, Šmilauer P. 2002. CANOCO v. 4.5, software for canonical community ordination. New York, NY: Microcomputer Power. library.wur.nl.

Trombulak SC, Frissell CA. 2000. Review of ecological effects of roads on terrestrial and aquatic communities. Conservation Biology, 14: 18-30. DOI: http://dx.doi.org/10.1046/j.15231739.2000.99084.x

WHO (The World Health Organization). 2013. Mortality and burden of disease from ambient air pollution. Access online: http:/www.who.int/gho/phe/en/. Retrieved 6/01/2015

Xiong $\mathrm{T}$, Leveque $\mathrm{T}$, Austruy A, Goix S, Schreck E, Dappe V, Sobanska S, Foucault Y, Dumat C. 2014. Foliar uptake and metal (loid) bioaccessibility in vegetables exposed to particulate matter. Environ. Geochem. Health, 36(5): 897-909. DOI: http://dx.doi.org/10.1007/s10653-0149607-6 